DOI $10.35381 / \mathrm{cm} \cdot v 5 i 1.280$

\title{
El Control interno como herramienta de apoyo a la gestión financiera del Gobierno Autónomo Descentralizado Municipal Zaruma
}

\section{Internal Control as a tool to support the financial management of the Zaruma Municipal Decentralized Autonomous Government}

\author{
Gianna Maritza Apolo Ordoñez \\ giannaapoloo@psg.ucacue.edu.ec \\ Universidad Católica de Cuenca, Cuenca \\ Ecuador \\ https://orcid.org/0000-0001-5176-8202 \\ Cecilia Ivonne Narváez Zurita \\ inarvaez@ucacue.edu.ec \\ Universidad Católica de Cuenca, Cuenca \\ Ecuador \\ https://orcid.org/0000-0002-7437-9880 \\ Juan Carlos Erazo Álvarez \\ jcerazo@ucacue.edu.ec \\ Universidad Católica de Cuenca, Cuenca \\ Ecuador \\ https://orcid.org/0000-0001-6480-2270
}

Recibido: 07 de septiembre de 2019

Aprobado: 28 de septiembre de 2019

\section{RESUMEN}

El control interno en las entidades gubernamentales del Ecuador es aplicado en base al ordenamiento jurídico vigente y a las normas de control interno emitidas por la Contraloría General del Estado. Sin embargo, al no existir procedimientos de control interno definidos en el departamento de gestión financiera del Gobierno Autónomo Descentralizado Municipal Zaruma se evidencian deficiencias de control, exponiéndose así a mayores riesgos financieros. Bajo este contexto el objetivo de la presente investigación es determinar la estructura de los procedimientos de control interno del área de gestión 


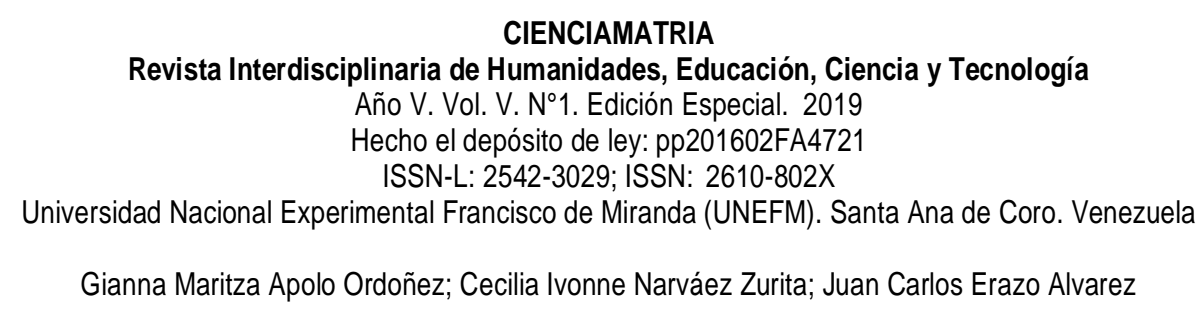

financiera con la finalidad de mejorar el control interno disminuyendo así la ocurrencia de posibles riesgos, además de generar información financiera veraz y confiable que contribuya a una correcta toma de decisiones. El diseño aplicado a la investigación fue no experimental y el enfoque que se le dio fue mixto.

Descriptores: Control interno; Riesgos; Recursos públicos; Toma de decisiones.

\begin{abstract}
The internal regulations of Ecuadorian government entities are applied based on a current judicial order and the internal norms of control as described by the Ecuadorian Government Accountability Office. However, as no defined procedures exist within the Department of Financial Processes of the Municipal Government of Zaruma, there is a lack of regulations, thus suggesting greater financial risks. Under this context, the objective of the present investigation is to determine the structure of these procedures the controls internal as they relate to financial processes, with the ultimate goal of improving the controls internal and thereby lowering the possibility of risks, as well as generating correct and reliable financial information that will allow for informed decision-making. The design of this investigation was not experimental and the approach that was taken was mixed-methods.
\end{abstract}

Descriptors: Internal control; Risks public resources; Decision making.

\title{
INTRODUCCIÓN
}

El control interno es una técnica administrativa utilizada por empresas privadas y públicas como medio de prevención ante posibles riesgos que afecten el desempeño de las actividades empresariales. El sistema de control interno se entiende como el conjunto de políticas, procedimientos y técnicas adoptadas desde la alta gerencia y por todo el personal de la organización y encaminadas al correcto funcionamiento de la empresa (Portal, 2016).

Actualmente en un mundo globalizado y con cambios tecnológicos, políticos, sociales y económicos permanentes, tanto el sector público y privado deben adaptarse y diseñar instrumentos que faciliten y mejoren el desarrollo de sus actividades, estos instrumentos 


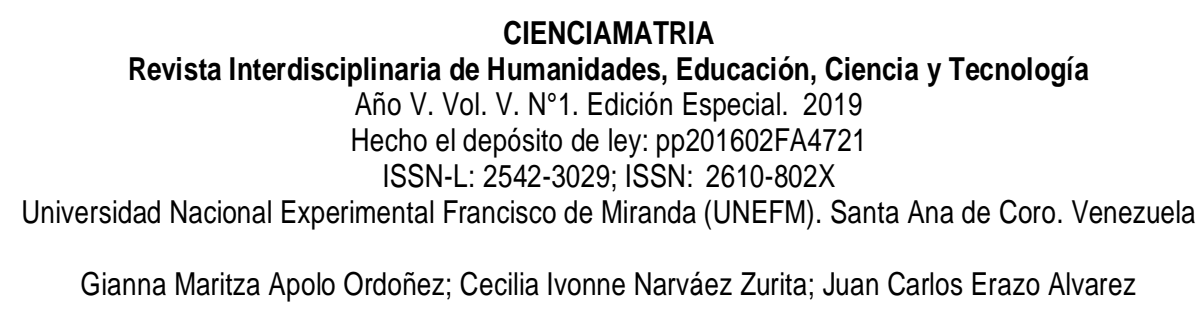

serán implementados como herramientas de administrativas de control, indispensables para la toma de decisiones y el logro de los objetivos institucionales (Estupiñan, 2015). En este contexto, el Gobierno Autónomo Descentralizado Municipal del cantón Zaruma no ha definido los procedimientos de control de las actividades que se realizan en el departamento de gestión financiera que permita el uso eficiente y eficaz de los recursos y garantice la veracidad y confiabilidad de la información, además al ser una institución pública se encuentra expuesta a mayores riesgos de fraudes financieros. Según López (2018) el sector público en los últimos años ha estado envuelto en mayores escándalos de corrupción, dejando al descubierto que no existe un control interno definido, conocido y aplicado, es decir carece de una estructura oportuna que establezca responsabilidades y tareas específicas al personal, así como del compromiso de las autoridades y directivos de las instituciones del sector público. Con base a esta problemática es necesario que la institución establezca políticas y procedimientos de control adecuados que faciliten el seguimiento a la gestión financiera y cumplimiento de los objetivos institucionales, Ortega Polanco (2017).

Un adecuado sistema de control interno transparentará el manejo de los recursos públicos y optimizará la prestación de servicios a la comunidad, evitando el incumplimiento de la ley y normativa vigente y protegiendo los recursos contra pérdidas, mala administración, errores, alteraciones y desfalcos, brindando de esta manera un grado de seguridad razonable para la toma de decisiones a la máxima autoridad y directores departamentales (Fonseca, 2011).

Lo antes expuesto, motiva a que en la presente investigación se establezcan procedimientos estructurados de control interno de los procesos financieros en el Gobierno Autónomo Descentralizado Municipal de Zaruma.

Fundamentos teóricos del control interno: con el fin de llevar un control de las actividades de las empresas y de corregir movimientos financieros erróneos nace el control interno como instrumento de vigilancia 


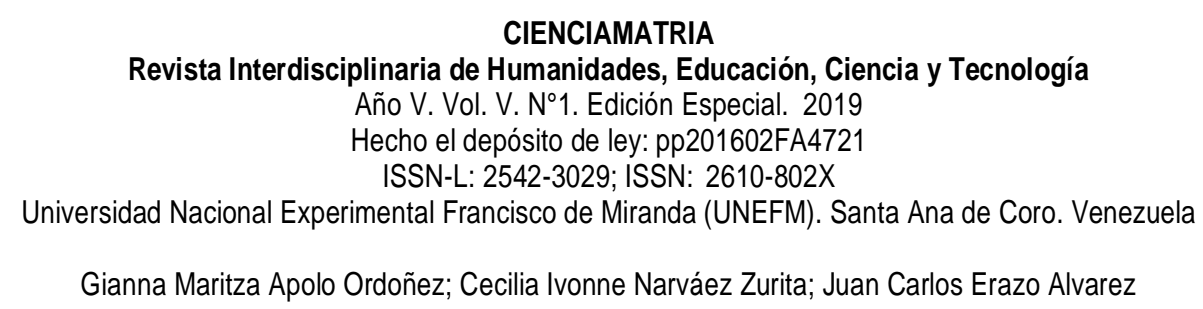

Los medios para vigilar la corrección de las operaciones financieras en las compañías, emergieron espontáneamente en la sociedad post-industrial. Una de las primeras herramientas que serviría para dicho cometido, sería el control interno desarrollado en la primera mitad del siglo XX (...). Los 90' fue una década de posicionamiento del control interno debido a que se divulgaron diversos estudios, entre ellos: Marco Integrado de Control Interno (Comité de Organizaciones patrocinadoras de la comisión Treadway), en 1992; Guía de control (Instituto de Contadores Autorizados de Canadá - CICA), en 1995; CobiT (Fundación de Sistemas de información, Auditoría y Control - ISACA), en 1996; y, el Marco de Referencia para los Sistemas de Control Interno en Organizaci9ones Bancarias (Comité de Basilea de Supervisión Bancaria), en 1998, tiempo después, se divulgó: Gestión de Riesgos Corporativos (ERM), en 2004; Guía COSO para Pequeñas Compañías Públicas, en 2006; y, la Guía de Monitoreo sobre Sistemas de Control Interno, en 2009. (Fonseca, 2011, p.12)

Como se observa el control interno nace como la necesidad de corregir las operaciones financieras erróneas de las empresas que traen como consecuencia el inadecuado uso de los recursos y fraudes financieros, a través del tiempo se han desarrollado diferentes estudios de control interno que se han ido ajustando a los cambios de la estructura organizativa, estrategias y nuevas tecnologías de las empresas.

Para que la investigación y estudio del sistema de control interno en el presente documento sean razonables, se abordarán los siguientes conceptos fundamentales El control interno es un proceso, ejecutado por la junta directiva o consejo de administración de una entidad, por su grupo directivo (gerencial) y por el resto del personal, diseñado específicamente para proporcionarles seguridad razonable de conseguir en la empresa las tres siguientes categorías: Efectividad y eficiencia de las operaciones, suficiencia y confiabilidad de la información financiera y cumplimiento de las leyes y regulaciones aplicables. (Estupiñan, 2015, pág. 27)

Para lograr la eficacia y eficiencia de las operaciones, es necesario que se definan los procedimientos que describan de manera clara las tareas encomendadas a cada uno de 


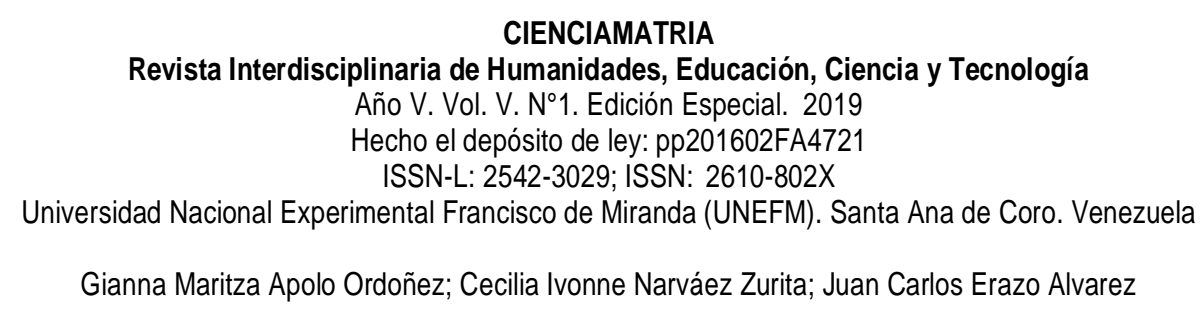

los servidores públicos del área de gestión financiera, las responsabilidades y sanciones que puede generar el incumplimiento de los procedimientos, Rodríguez Rodríguez, Crúz \& Puertas (2017).

Para que la empresa tenga la certeza de que la información financiera es fiable, la comunicación es un factor determinante, para lograrlo se deberá diseñar y consolidar una técnica adecuada de comunicación entre los departamentos que facilite el intercambio de información, además de definir indicadores que evalúen el cumplimiento de este objetivo. Cumplimiento de las Leyes y normas aplicables, para que el sistema de control interno sea eficiente, el conocimiento de las leyes y normativa es requisito indispensable y obligatorio tanto para el director y personal del área financiera de la empresa, si existe desconocimiento de la normativa y de la importancia del sistema de control interno en la empresa, este fracasará.

En este contexto, el sistema de control interno debe ser implementado como un proceso sistemático y adaptado de acuerdo a la naturaleza de las empresas como medio para corregir las operaciones financieras erróneas que pueden conllevar un incorrecto uso de los recursos y posibles fraudes financieros (Contraloría General de la República, 2014). Según Barquero (2013), Cámara de Diputados (2014), Vega y Ortiz (2017), Mayorga y Llagua (2018) para que un sistema de control interno sea adecuado y fortalezca a las organizaciones, se deben definir los procedimientos, métodos y reglamentación institucional con base a la normativa y legislación vigente, respaldando así una correcta toma de decisiones de los directivos para una administración eficiente. Sin embargo, la implementación de un sistema de control interno no evita que la empresa se encuentre expuesta a errores financieros.

Para Fonseca (2011), Contraloría General del Estado (2014) y Vega (2016) los directivos de la empresa tendrán el compromiso de vigilar que el personal cumpla con las responsabilidades asignadas a cada uno, así como fomentar un seguimiento y evaluación de actividades permanente con el fin de evitar errores que debiliten la empresa y se exponga a riesgos. Dentro de este contexto, la relación entre los directivos y empleados 


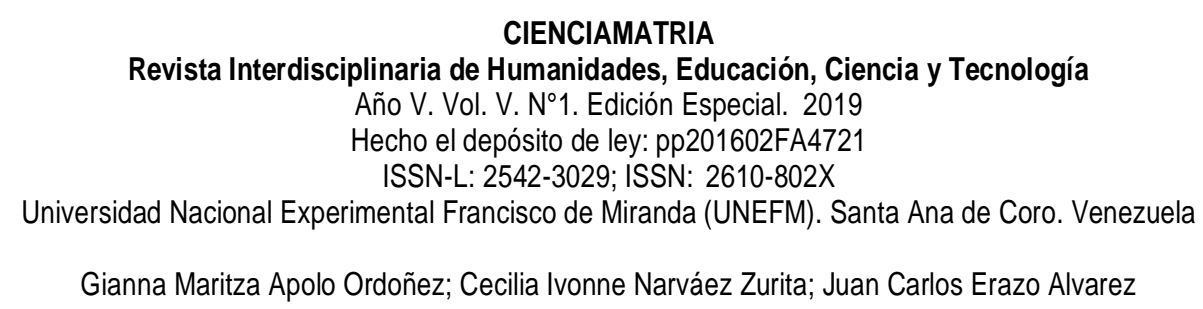

de la empresa debe ser sólida, de esta manera el personal se verá involucrado en el proceso de recolección y proceso de la información, y serán capaces de proponer planes o programas de mejoramiento que contribuyan a un exitoso sistema de control interno. Romero (2013) y Estupiñan (2015), definen las características del control interno como: es un proceso; es decir, un medio para lograr un fin y no un fin en sí mismo, lo llevan a cabo las personas que actúan en todos los niveles y no se trata solamente de manuales de organización y procedimientos, en cada área de la organización, el funcionario encargado de dirigirla es responsable por el control interno ante su jefe inmediato de acuerdo con los niveles de autoridad establecidos; y en su cumplimiento participan todos los trabajadores de la entidad independientemente de su categoría ocupacional. Aporta un grado de seguridad razonable, con relación al logro de los objetivos fijados; no la total, debe facilitar la consecución de objetivos en una o más de las áreas u operaciones en la entidad, debe propender al logro del autocontrol, liderazgo y fortalecimiento de la autoridad y responsabilidad de los colectivos.

Modelos de control interno: de manera general se abordará los tres principales modelos de control interno que han sido desarrollados y adaptados en Latinoamérica con el fin de contribuir al éxito de las empresas, a partir de una mayor participación de gerentes, directivos y personal.

El control interno según COSO es el conjunto de acciones interrelacionadas que ejecutan todos los integrantes de las organizaciones encaminadas a alcanzar las metas y objetivos planteados, evitar pérdidas financieras, información financiera confiable y una correcta aplicación de las leyes y normas (Estupiñan, 2015).

El control interno según el modelo COCO es implementado por la alta gerencia a través de un lenguaje más simple y accesible para todos los empleados contribuyendo así a la toma de decisiones por medio de una mejor comprensión del control, el riesgo y la dirección (Fonseca, 2011).

El modelo MICIL (Marco Integrado de Control Interno para Latinoamérica), surge tras la necesidad de un brindar un enfoque adecuado a la situación de Latinoamérica, según 


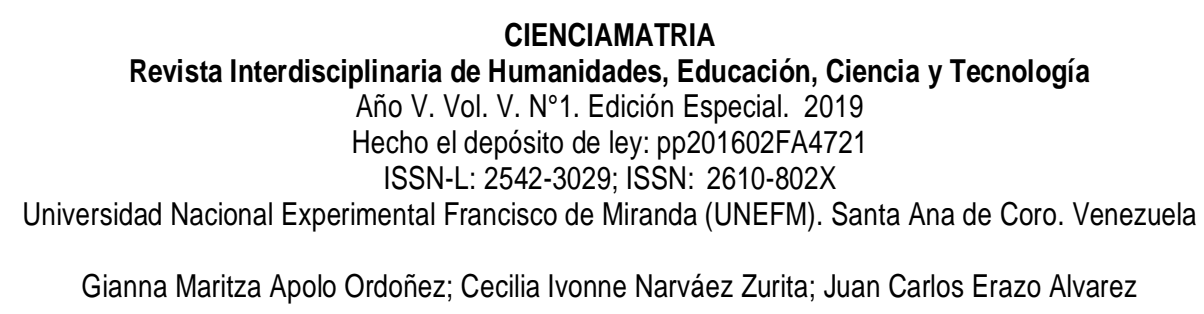

este modelo el control interno es ejecutado por los empleados y servidores de la organización a las actividades de la empresa y su finalidad es generar cierto grado de seguridad razonable a las operaciones ejecutadas (Casals y Associates, Inc., 2004). Componentes del sistema de control interno en base a COSO I: para Romero (2013) y Estupiñan (2015) los elementos del sistema de control interno son cinco, los mismos que se relacionan entre si y son inherentes a la práctica de gestión de la empresa.

Ambiente de control: según Escalante (como se citó en Serrano, Señalin, Vega, y Herrera, 2018) este componente es la base sobre el que actúan los otros componentes de control, se basa en la estructura organizativa de la empresa, la reglamentación interna, el compromiso de la alta gerencia, directivos y el resto de personal para el logro de los objetivos y metas trazadas por la empresa, el liderazgo, los valores éticos y la planificación son el eje de este componente. Se puede definir que los factores del ambiente de control son: Integridad y valores éticos,

- Competencia profesional,

- Actividades de la junta directiva y el comité de auditoría,

- Estilo de gerencia,

- Estructura organizacional, delegación y responsabilidades,

- Políticas y prácticas de talento humano.

Evaluación de riesgos: para la Contraloría General de la República (2014) la evaluación de riesgos es la identificación, análisis y valoración de los riesgos que puedan generarse en las labores cotidianas de la empresa y que pueden ser una limitante para el logro de los objetivos, la evaluación de riesgos es además la base para determinar la forma en que tales riesgos deben ser administrados. Por medio de la gerencia se establecen los objetivos generales y específicos, paralelamente se identifican y analizan los riesgos de que dichos objetivos no se logren o afecten el óptimo uso de sus bienes y recursos, mantener ventajas competitivas, mantener la imagen empresarial, incrementar y mantener su solidez financiera y mantener su progreso. El 


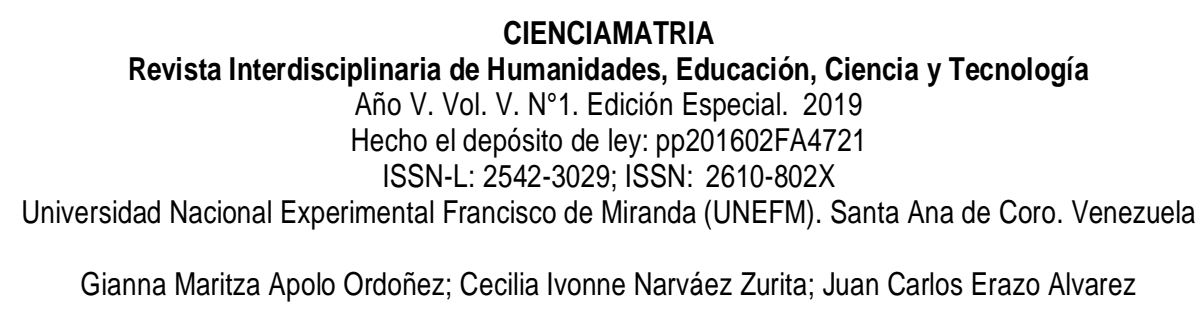

proceso en el análisis de riesgos se realiza a través de: la estimación de la importancia del riesgo y sus efectos, la evaluación de la probabilidad de ocurrencia, el establecimiento de acciones y controles necesarios y la evaluación periódica del proceso anterior (Estupiñan, 2015).

Actividades de control: Son aquellas que realizan la gerencia y demás personal de la organización para cumplir diariamente con las actividades asignadas. Estas actividades están expresadas en las políticas, sistemas y procedimientos. Los tipos de control aplicables son los siguientes: defectivos, preventivos y correctivos (Contraloría General del Estado, 2014).

Sistemas de información y comunicación: para poder controlar una entidad y tomar decisiones correctas respecto a la obtención, uso y aplicación de los recursos, es necesario disponer de información adecuada y oportuna. La información pertinente debe ser identificada, capturada y comunicada al personal en la forma y dentro del tiempo indicada, lo que permitirá el cumplimiento de sus responsabilidades. Las principales características de este componente son: oportunidad, actualización, razonabilidad y accesibilidad (Estupiñan, 2015).

Supervisión y monitoreo: la gerencia debe llevar a cabo la revisión y evaluación sistemática de los componentes y elementos que forman parte de los sistemas de control. La evaluación debe conducir a la identificación de los controles débiles, insuficientes o innecesarios, para promover su robustecimiento e implantación. Los niveles de control están clasificados de la siguiente manera: control de alto nivel, controles específicos de nivel inferior, controles específicos de nivel superior, control de nivel inferior (Estupiñan, 2015).

Se puede observar que en la mayoría de la literatura revisada los autores coinciden en que un oportuno sistema de control interno no debe ser un proceso aislado en las organizaciones, es importante el involucramiento de todo el personal, partiendo desde los directivos quienes serán los encargados de determinar los métodos, técnicas y 


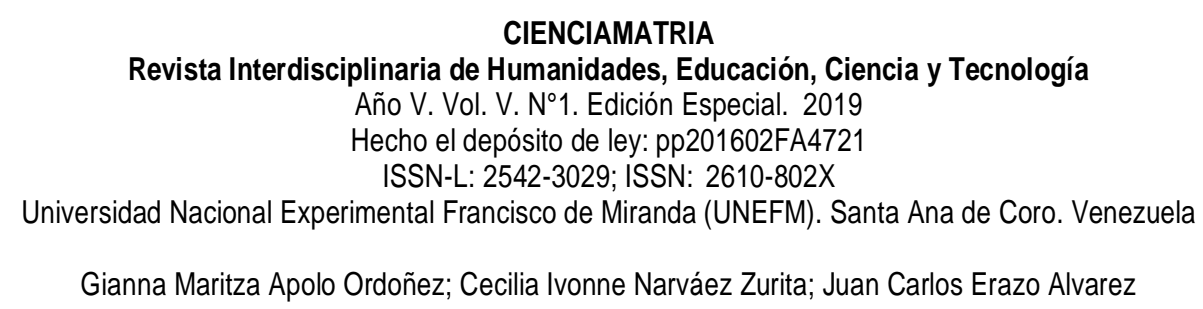

procedimientos de control, mientras que el resto del personal será responsable de la aplicación, seguimiento y evaluación de resultados.

\section{SISTEMA DE CONTROL INTERNO EN EL SECTOR PÚBLICO}

En los apartados anteriores se analizó la importancia del sistema de control interno en cualquier tipo de organización, sea pública o privada, este epígrafe está orientado a analizar el sistema de control interno en el sector público, entendiéndolo como aquel que está integrado por el ejecutivo, legislativo y por todas las instituciones públicas y personas jurídicas de derecho privado que manejen recursos públicos.

Según la Asamblea Constituyente (2008) el sector público en el Ecuador está conformado por Ministerios y otras instituciones del Estado, los Gobiernos Autónomos Descentralizados, las Empresas Públicas, la Banca Pública y las instituciones de la Seguridad Social; todas estas prestan bienes y servicios a la sociedad conociéndoselas como: sector público. El objetivo fundamental de estas instituciones es el bienestar social, haciéndose necesaria una gestión eficiente, efectiva y responsable; en este sentido, el sistema de control interno es una herramienta administrativa indispensable para alcanzar los objetivos y las metas en base a la aplicación de disposiciones vigentes que a su vez consoliden la transparencia de la gestión.

Actualmente en el Ecuador existen 1042 Gobiernos Autónomos Descentralizados organizados en: 24 provincias, 1 distrito metropolitano, 220 municipios y 797 juntas parroquiales, según la (Asamblea Constituyente, 2008) en la Constitución de la República del Ecuador Art. 238.- Los gobiernos autónomos descentralizados gozarán de autonomía política, administrativa y financiera (...). El concepto de descentralización y autonomía planteado por la Constitución del 2008 representa un desafío para los Gobiernos Autónomos Descentralizados que deben asumir competencias exclusivas que permitan mejorar la calidad de vida de los habitantes de sus comunidades.

En los últimos años en Ecuador se ha dejado al descubierto el deficiente sistema de control de las entidades públicas, reflejándose en un sin número de escándalos de 
corrupción (López, 2018), todo esto a pesar de que en la Constitución de la República del Ecuador en su artículo 211 instituye a la Contraloría General del Estado como el órgano de control encargado del buen uso de los recursos públicos y el cumplimiento de los objetivos de los organismos estatales y de las personas jurídicas de derecho privado que manejen recursos públicos,

La Contraloría General del Estado ha sido la encargada de emitir las normas de control interno que rigen y son de cumplimiento obligatorio para todas las instituciones del Estado. La Contraloría General del Estado (2014) refiere que: las instituciones del Estado que manejen recursos públicos tendrán bajo su responsabilidad el control interno, para ello definirán los procesos, técnicas y procedimientos a implementarse desde la máxima autoridad, la dirección y los servidores públicos, logrando así seguridad razonable en el desarrollo de las actividades, un correcto control de los recursos públicos y el logro de los objetivos institucionales.

Las normas de control interno aplicables en los organismos gubernamentales han sido realizadas con base a los cinco componentes COSO I, ambiente de control, evaluación de riesgos, actividades de control, información y comunicación y seguimiento.

Sin embargo, si se analiza la situación actual del sistema público es fácil percibir el incipiente sistema de control implementado, la falta de compromiso de los altos funcionarios, integridad y valores éticos incipientes y procedimientos no establecidos han generado una serie de errores e irregularidades de los servidores públicos quedando en estado de vulnerabilidad los activos del sector público.

\section{GESTIÓN FINANCIERA}

La gestión financiera es el eje principal para el éxito de las empresas, una gestión financiera eficiente y eficaz tiene como objetivos principales la mejora continua de la empresa con el fin de mantenerse a la vanguardia con los cambios que exige el entorno, crecer y mantener la competitividad en el mercado, reducir los niveles de riesgo que 


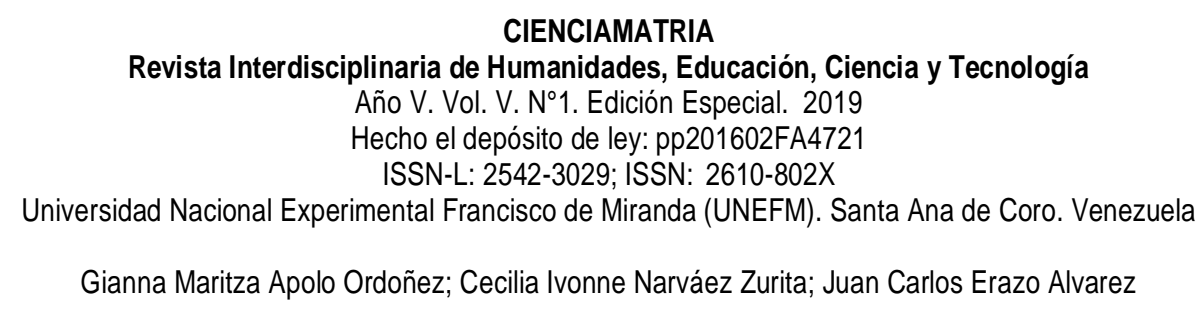

obstaculicen su posicionamiento en el mercado y finalmente satisfacer a los stakeholder (Perez y Carvallo, 2015).

En un mundo globalizado con cambios permanentes el gerente financiero debe adoptar estrategias como medio para maximizar los beneficios de la empresa y el correcto manejo de los recursos económicos, una apropiada toma de decisiones llevará al éxito de la empresa, al contrario, una toma de decisiones errónea ocasionará el deterioro de la organización, llevándola incluso al colapso (Córdoba, 2012).

Para Córdoba (2012) la gestión finanicera esta estrechamente relacionada con la toma de decisiones, la información generada por las empresas será la herramienta fundamental para los directivos en la toma de decisiones financieras, la información contable de manera particular es elemental, los estados financieros de las instituciones reflejan de manera precisa la situación económico - financiera que atraviesan, sea está consolidada y solvente o débil y de riesgo.

Según Mendoza (2013) la gestión financiera en el ambito público es limitada, esto es propiciado por los escazos recursos financieros asignados para atender un aparato burocrático creciente, a diferencia de la gestión financiera privada la púbica esta orientada a maximizar el beneficio de la comunidad, mediante la inversión en obras y servicios que mejoren la calidad de vida de los individuos, esto no siempre esta reflejado en los estados financieros, la mayoría de los beneficios generados a la comunidad no son cuantificables monetariamente.

Los instrumentos de la gestión finanicera pública están establecidos como: plan operativo anual, plan anual de contratación, planificación plurianual, plan de desarrollo y ordenamiento territorial, presupuesto, planificación financiera, plan de desarrollo y ordenamiento territorial (Mendoza, 2013).

De todos los instrumentos utilizados en la gestión financiera pública el de mayor relevancia e importancia es el presupuesto, esto no quiere decir que los demás no sean importantes, simplemente se ubican en una escala menor (Mendoza, 2013). 


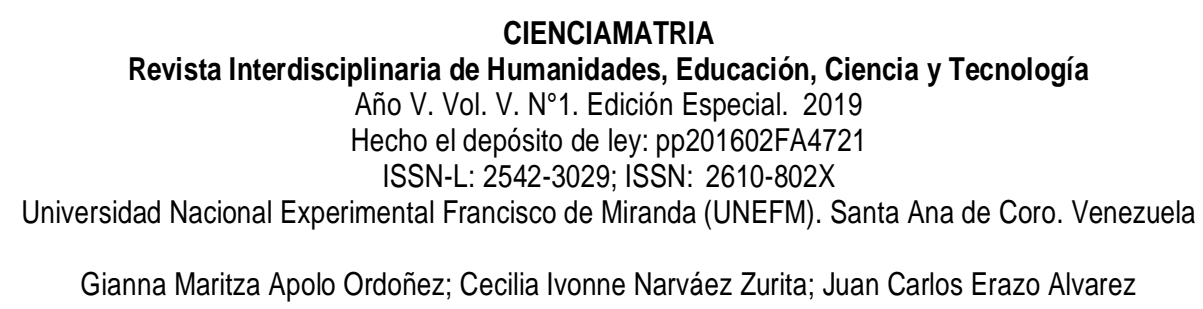

Del análisis realizado se concluye, que para que una gestión financiera sea eficiente es indispensable la utilización de herramientas de control interno que contribuyan a optimizar los recursos financieros, evitar posibles fraudes y controlar que las actividades de los servidores públicos se enmarquen en principios de ética y transparencia.

\section{METODOLOGÍA}

El diseño aplicado en la presente investigación fue no experimental, esto debido a que las variables de estudio no se expusieron a ningún tipo de manipulación, el enfoque que se le dio a la investigación fue mixta, es decir la combinación de los métodos cualitativo y cuantitativo, como primer paso se analizó la literatura sobre el tema de control interno, el siguiente paso fue la determinación de resultados en base a un diagnóstico realizado a la institución. Respecto al alcance de la presente investigación ésta fue descriptiva ya que se detallaron las particularidades de la institución y de los servidores que laboran en el Gobierno Autónomo Descentralizado Municipal de Zaruma.

Los métodos que se aplicaron fueron: inductivo - deductivo, histórico lógico, analítico sintético y estadístico, según la finalidad el estudio fue transversal lo que permitió analizar el comportamiento de los servidores públicos en el desempeño de sus funciones en un determinado momento de tiempo, las variables se midieron a través de las técnicas de la encuesta y la entrevista. En la primera técnica se estableció un cuestionario de preguntas cerradas, mientras que en la entrevista se generaron preguntas abiertas y ésta fue dirigida a la máxima autoridad de la institución. Para llevar adelante la investigación se encuestó al $100 \%$ de la población involucrada en el desempeño de actividades del departamento financiero, por tanto, no se procedió a realizar muestreo (Hernández, Fernández y Baptista, 2006).

\section{RESULTADOS}

Una vez que se aplicaron los instrumentos de investigación se obtuvieron los siguientes resultados. 


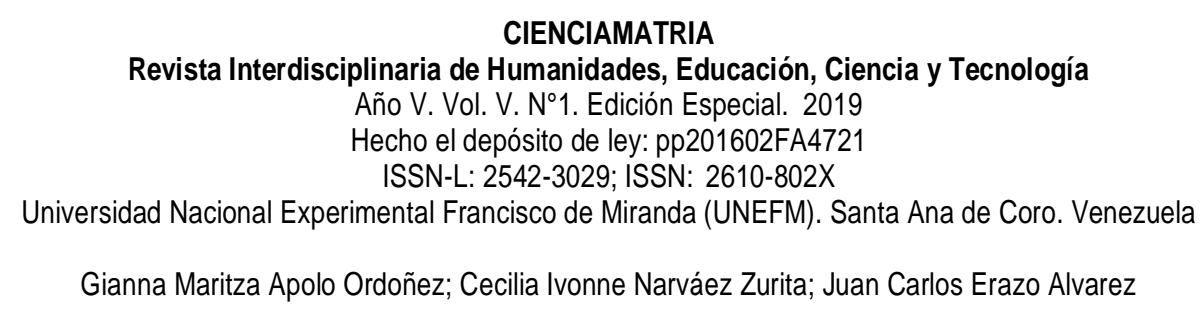

Reglamento orgánico funcional por procesos: el reglamento orgánico funcional por procesos constituye el pilar fundamental en la determinación de responsabilidades de cada uno de los servidores públicos, sin embargo, se encuentra desactualizado, además el que está vigente no ha sido socializado y la mayoría de los servidores públicos no conocen las funciones que deben desempeñar en cada uno de sus puestos de trabajo. Nivel de competencia profesional: es importante que el personal de la institución mantenga su nivel de competencia por lo que la unidad de talento humano realizará el plan de capacitación de personal de acuerdo a las necesidades de los servidores, en el GAD Municipal de Zaruma no existen programas de capacitación permanente al personal, los servidores del departamento de gestión financiera asisten de manera esporádica a capacitaciones de otros organismos gubernamentales como: Banco de Desarrollo y Asociación de Municipalidades Ecuatorianas (AME), la escasa capacitación al personal ha contribuido a un menor nivel de competencia profesional.

Unidad de auditoría de control interno: la auditoría interna es desarrollada de manera independiente se enfoca en mejorar las actividades de una empresa para el logro de los objetivos. Actualmente en el Gobierno Autónomo Descentralizado Municipal del cantón Zaruma no existe una unidad de auditoría interna, lo que conlleva a una mayor probabilidad de cometer errores y estar expuesto a riesgos financieros.

Identificación de riesgos: este permite identificar los posibles eventos potenciales de riesgos que puedan obstaculizar el cumplimiento de los objetivos de la entidad, pudiendo ser internos o externos. Los riesgos internos son los que se encuentran bajo el control de la institución, mientras que los externos se encontrarán fuera del control institucional.

El departamento de gestión financiera no ha identificado las procedencias de posibles eventos de riesgo, las causas que los generarían y los impactos sobre los objetivos y metas institucionales.

Plan de mitigación de Riesgos: una vez identificados los riesgos, se definirán acciones que deben ser detalladas en el plan de mitigación de riesgos, estas deberán ser 


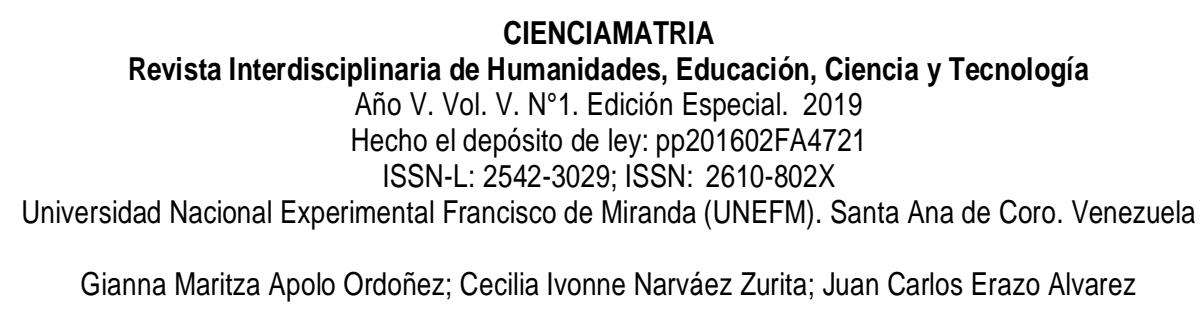

redactadas de manera clara y precisa con el fin de que sean entendidas por todos los servidores públicos para una respuesta oportuna en caso de alguna ocurrencia de riesgo. El departamento de gestión financiera al no haber identificado los riesgos, no ha generado un plan de mitigación de riesgos.

Rotación de labores: La rotación de labores contribuye a reducir riesgos, los directivos de la empresa a través del orgánico funcional definirán las funciones de cada servidor, y en lo posible rotarán funciones entre quienes realicen actividades afines.

Las funciones de cada uno de los servidores del área financiera del GAD Municipal de Zaruma se encuentran definidas en el manual orgánico funcional de la institución, sin embargo, no todos conocen las actividades que deben desarrollar de acuerdo al perfil de su puesto.

Procedimientos de control del área financiera: los procedimientos se definen como los métodos a seguir para el logro de objetivos y metas propuestas por la institución, bajo este enfoque se puede deducir que los procedimientos de control a los procesos que se generan en el área de gestión financiera no se han definido lo que conlleva a un mayor índice de probabilidades de errores y a que se presenten deficiencias de control que limitaran el cumplimiento de los objetivos planteados que posteriormente serán observados por el organismo de control que es la Contraloría General del Estado.

Políticas y procedimientos de archivo: Los archivos físicos y digitales deben establecerse de acuerdo a estándares definidos por la empresa y en concordancia con la normativa legal vigente, esto con el fin de mantener y preservar la información, la misma que estará disponible para verificaciones futuras. Una falencia en el departamento financiero es la carencia de un sistema de archivo físico y digital bajo normas técnicas y jurídicas, el archivo que se maneja es físico y no responde a las necesidades del área.

Información y comunicación: los sistemas de información facilitan el registro, proceso e información sobre las operaciones del área financiera, estos sistemas deberán estar diseñados e implementados de acuerdo a las necesidades de la organización. Al analizar 
el sistema de información del área financiera se puede apreciar que no se ha desarrollado un sistema de información que se ajuste a las necesidades del departamento.

Con base al análisis de los resultados obtenidos se propone definir los procedimientos de control interno a los procesos que se desarrollan en el área financiera como herramienta de apoyo para reducir la ocurrencia de riegos financieros. En la figura 1 se aprecia la estructura de la propuesta, la misma que contempla los procedimientos de control para las áreas: presupuesto, tesorería, recaudación, contabilidad y deuda pública.

\section{Figura 1}

\section{Procedimientos de control interno}

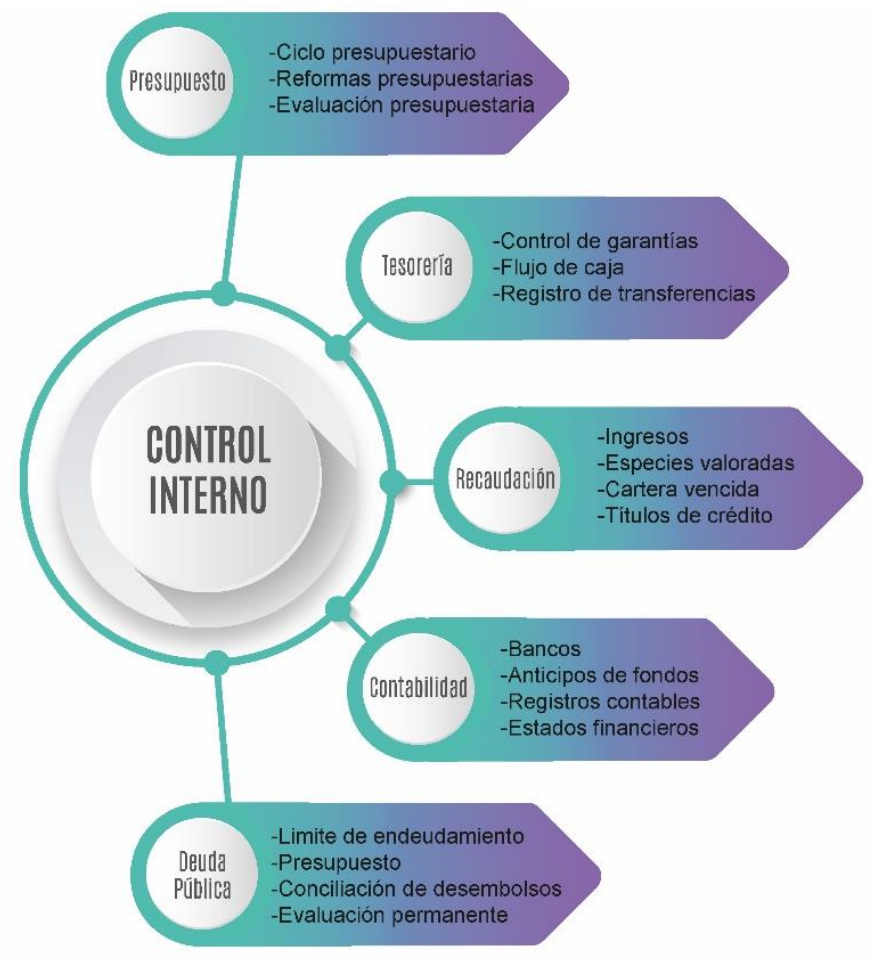

Los procedimientos de control interno propuestos integran un conjunto de instrucciones, directrices y actividades considerando principios de eficiencia, eficacia y economía encaminados a determinar la razonabilidad, en cuanto a la ejecución de los procesos financieros del GAD Municipal de Zaruma. 
Revista Interdisciplinaria de Humanidades, Educación, Ciencia y Tecnología

Año V. Vol. V. №1. Edición Especial. 2019

Hecho el depósito de ley: pp201602FA4721

ISSN-L: 2542-3029; ISSN: 2610-802X

Universidad Nacional Experimental Francisco de Miranda (UNEFM). Santa Ana de Coro. Venezuela

Gianna Maritza Apolo Ordoñez; Cecilia Ivonne Narváez Zurita; Juan Carlos Erazo Alvarez

A continuación, se detallan los procedimientos de control, objetivos, acciones de control y evidencias.

\section{Tabla 1}

Presupuesto

Gobierno Autónomo Descentralizado Municipal de Zaruma

\section{Procedimientos de control interno}

\section{Cumplimiento del ciclo presupuestario}

\begin{tabular}{|c|c|c|c|c|}
\hline $\begin{array}{l}\mathbf{N} \\
\underline{0}\end{array}$ & Procedimiento & Objetivo & Acciones de control & Evidencia \\
\hline 1 & \multirow[t]{2}{*}{$\begin{array}{l}\text { Revisión de } \\
\text { cumplimiento de la } \\
\text { programación } \\
\text { presupuestaria. } \\
\text { Responsable: } \\
\text { Director del } \\
\text { Departamento de } \\
\text { Gestión de } \\
\text { Planificación }\end{array}$} & $\begin{array}{l}\text { Definir los programas, } \\
\text { proyectos y } \\
\text { actividades que se } \\
\text { incorporaran en el } \\
\text { presupuesto. }\end{array}$ & $\begin{array}{l}\text { Verificar que la } \\
\text { programación } \\
\text { presupuestaria se } \\
\text { encuentre alineada al } \\
\text { plan nacional de } \\
\text { desarrollo y de } \\
\text { ordenamiento territorial. }\end{array}$ & $\begin{array}{l}\text { Informe del } \\
\text { responsable del } \\
\text { departamento de } \\
\text { planificación y } \\
\text { ordenamiento } \\
\text { territorial. }\end{array}$ \\
\hline & & & $\begin{array}{l}\text { Constatar que la } \\
\text { programación } \\
\text { presupuestaria se } \\
\text { enmarque dentro de las } \\
\text { disponibilidades } \\
\text { presupuestarias. }\end{array}$ & $\begin{array}{l}\text { Informe de } \\
\text { estimación de } \\
\text { ingresos emitido } \\
\text { por el } \\
\text { departamento de } \\
\text { gestión } \\
\text { financiera. }\end{array}$ \\
\hline
\end{tabular}


Revista Interdisciplinaria de Humanidades, Educación, Ciencia y Tecnología

Año V. Vol. V. №1. Edición Especial. 2019

Hecho el depósito de ley: pp201602FA4721

ISSN-L: 2542-3029; ISSN: 2610-802X

Universidad Nacional Experimental Francisco de Miranda (UNEFM). Santa Ana de Coro. Venezuela

Gianna Maritza Apolo Ordoñez; Cecilia Ivonne Narváez Zurita; Juan Carlos Erazo Alvarez

2 Verificación de que

en la proforma

presupuestaria se

encuentra plasmada

la primera fase del

ciclo

presupuestario.

Responsable:

Director de Gestión

Financiera
Controlar que la

proforma

presupuestaria se

encuentre elaborada

de acuerdo a las

directrices, catálogos

y clasificadores

presupuestarios

emitidos por el

Ministerio de

Economía y Finanzas.
Controlar que en todos

los programas se

establezcan objetivos,

metas, recursos

necesarios y plazos de

ejecución.

Confirmar que la

programación se la

realizó de forma

participativa de

acuerdo a lo

establecido por la

Constitución y la ley.

Comparar las partidas

presupuestarias de la

proforma con las del

clasificador

presupuestario emitido

por el Ministerio de

Economía y Finanzas

Evidenciar que los

ingresos y egresos que

consten en la proforma

presupuestaria se

encuentren justificados.
POA institucional y cronograma con

firmas de

responsabilidad.
Hojas de asistencia.

Resolución de aprobación del concejo de participación ciudadana. Informe de verificación emitido por el área de presupuesto.

POA suscrito por directores departamentales.
3 Comprobación de que el presupuesto institucional ha sido aprobado en los plazos previstos en la ley.

Responsable:

Director de Gestión

Financiera
Verificar que la fase

de programación, formulación y aprobación presupuestaria se encuentre dentro de los plazos establecidos en la ley.
Revisión de oficios

cursados, informes de

reuniones con la

comunidad, informes

entregados a la máxima autoridad y concejo Municipal.
Oficios, informes, resolución de aprobación. 


\author{
CIENCIAMATRIA \\ Revista Interdisciplinaria de Humanidades, Educación, Ciencia y Tecnología \\ Año V. Vol. V. №1. Edición Especial. 2019 \\ Hecho el depósito de ley: pp201602FA4721 \\ ISSN-L: 2542-3029; ISSN: 2610-802X \\ Universidad Nacional Experimental Francisco de Miranda (UNEFM). Santa Ana de Coro. Venezuela \\ Gianna Maritza Apolo Ordoñez; Cecilia Ivonne Narváez Zurita; Juan Carlos Erazo Alvarez
}

$4 \quad$ Verificación de que

el presupuesto

aprobado entró en

vigencia el 01 de

enero y concluyó el

31 de diciembre.

Responsable:

Director de Gestión

Financiera
$5 \quad$ Verificación de que los recursos

materiales y

financieros han

sido utilizados de

acuerdo a lo

previsto en la

proforma

presupuestaria.

Responsable:

Analista de

presupuesto.

6 Comprobación de

que el presupuesto

ha sido clausurado

el 31 de diciembre.

Responsable:

Contador General
Confirmar que el presupuesto entró en vigencia a partir del 01 de enero.
Revisar el sistema SIG-

AME.

Registro

presupuestario

inicial.
Ratificar que la clausura del presupuesto se ejecutó de acuerdo a lo estipulado en la ley.
Garantizar que los bienes, servicios recibidos de acuerdo a lo establecido por la institución
Confirmar que el presupuesto aprobado ha sido enviado al Ministerio de Finanzas en el plazo establecido por la normativa vigente.

Comprobar que dentro del proceso de ejecución presupuestaria existan los documentos justificativos y comprobatorios que demuestren la entrega de obras, servicios y bienes contratados.

Oficio dirigido al Ministerio de Finanzas. Guía de remisión.

Informe de ejecución presupuestaria. Informe de necesidad institucional. Certificación presupuestaria. Facturas. Actas entrega recepción.

Revisión de registros contables y estados financieros.

Informe de contabilidad.

Estados financieros Informe de ejecución presupuestaria

\title{
Tabla 2
}

Tesorería

\section{Gobierno Autónomo Descentralizado Municipal de Zaruma}


Revista Interdisciplinaria de Humanidades, Educación, Ciencia y Tecnología

Año V. Vol. V. №1. Edición Especial. 2019

Hecho el depósito de ley: pp201602FA4721

ISSN-L: 2542-3029; ISSN: 2610-802X

Universidad Nacional Experimental Francisco de Miranda (UNEFM). Santa Ana de Coro. Venezuela

Gianna Maritza Apolo Ordoñez; Cecilia Ivonne Narváez Zurita; Juan Carlos Erazo Alvarez

Procedimientos de control interno

\section{Conservación y protección de garantías}

\begin{tabular}{|c|c|c|c|c|}
\hline № & Procedimiento & Objetivo & Acciones de control & Evidencia \\
\hline \multirow[t]{2}{*}{1} & $\begin{array}{l}\text { Confirmación de } \\
\text { que las garantías } \\
\text { cumplan con las } \\
\text { condiciones } \\
\text { contempladas en la } \\
\text { ley, convenios y } \\
\text { contratos. } \\
\text { Responsable: } \\
\text { Tesorero }\end{array}$ & $\begin{array}{l}\text { Comprobar que previo } \\
\text { a la entrega de } \\
\text { anticipos, las } \\
\text { garantías sean } \\
\text { entregadas a } \\
\text { tesorería cumpliendo } \\
\text { con lo establecido en } \\
\text { la legislación vigente. }\end{array}$ & $\begin{array}{l}\text { Se procede a revisar } \\
\text { que los montos de las } \\
\text { garantías se } \\
\text { encuentren de acuerdo } \\
\text { a los montos } \\
\text { establecidos por la ley. }\end{array}$ & $\begin{array}{l}\text { Contratos } \\
\text { suscritos entre el } \\
\text { GAD Municipal y } \\
\text { proveedores. } \\
\text { Pólizas. }\end{array}$ \\
\hline & & & $\begin{array}{l}\text { Constatar la vigencia y } \\
\text { vencimiento de las } \\
\text { garantías }\end{array}$ & \\
\hline 2 & $\begin{array}{l}\text { Verificación de la } \\
\text { vigencia de las } \\
\text { garantías. } \\
\text { Responsable: } \\
\text { Tesorero }\end{array}$ & $\begin{array}{l}\text { Confirmar que las } \\
\text { garantías se } \\
\text { encuentran vigentes. }\end{array}$ & $\begin{array}{l}\text { Constatar que tesorería } \\
\text { ha informado a su } \\
\text { inmediato superior } \\
\text { sobre el vencimiento de } \\
\text { las garantías para el } \\
\text { trámite de renovación o } \\
\text { ejecución. } \\
\text { del flujo de caja }\end{array}$ & $\begin{array}{l}\text { Informes } \\
\text { entregados. }\end{array}$ \\
\hline 1 & $\begin{array}{l}\text { Comprobación de } \\
\text { que el flujo de caja } \\
\text { se ha realizado de } \\
\text { acuerdo a las } \\
\text { entradas y salidas } \\
\text { de fondos. } \\
\text { Responsable: } \\
\text { Tesorero }\end{array}$ & $\begin{array}{l}\text { Verificar que la } \\
\text { programación de flujo } \\
\text { de caja responda a la } \\
\text { disponibilidad real de } \\
\text { fondos. }\end{array}$ & $\begin{array}{l}\text { Se procede a constatar } \\
\text { que exista una } \\
\text { programación de flujo } \\
\text { de caja. } \\
\text { Se revisa que la } \\
\text { programación sea } \\
\text { realizada siguiendo } \\
\text { una periodicidad. } \\
\text { Revisión de las cedulas } \\
\text { presupuestarias de } \\
\text { ingresos. } \\
\text { Revisión de los } \\
\text { ingresos a las cuentas } \\
\text { bancarias del GAD } \\
\text { Municipal. }\end{array}$ & $\begin{array}{l}\text { Plan de } \\
\text { programación } \\
\text { aprobado. } \\
\text { Cedulas } \\
\text { presupuestarias } \\
\text { de ingresos. } \\
\text { Estados de } \\
\text { cuenta. }\end{array}$ \\
\hline
\end{tabular}

Control previo al registro de transferencias 
CIENCIAMATRIA

Revista Interdisciplinaria de Humanidades, Educación, Ciencia y Tecnología

Año V. Vol. V. №1. Edición Especial. 2019

Hecho el depósito de ley: pp201602FA4721

ISSN-L: 2542-3029; ISSN: 2610-802X

Universidad Nacional Experimental Francisco de Miranda (UNEFM). Santa Ana de Coro. Venezuela

Gianna Maritza Apolo Ordoñez; Cecilia Ivonne Narváez Zurita; Juan Carlos Erazo Alvarez

1 Validación de la documentación presentada para generar la transferencia de fondos.

Responsable:

Director de Gestión

Financiera

Elaborado por:
Verificar que la información entregada previa a la transferencia de fondos sea confiable y valida.
Se revisa que exista la documentación suficiente y pertinente. Se examina que la documentación se encuentre legalizada con firmas de responsabilidad.

Revisado por:

Tabla 3

Recaudación

Gobierno Autónomo Descentralizado Municipal de Zaruma

\author{
Procedimientos de control interno
}

Verificación de ingresos

\begin{tabular}{|c|c|c|c|c|}
\hline № & Procedimiento & Objetivo & Acciones de control & Evidencia \\
\hline 1 & $\begin{array}{l}\text { Revisión de las } \\
\text { cajas recaudadoras. } \\
\text { Responsable: } \\
\text { Tesorero }\end{array}$ & $\begin{array}{l}\text { Controlar que la } \\
\text { recaudación se esté } \\
\text { realizando de acuerdo } \\
\text { al ordenamiento } \\
\text { jurídico vigente. }\end{array}$ & $\begin{array}{l}\text { Arqueos sorpresivos de } \\
\text { caja. } \\
\text { Verificar que los } \\
\text { ingresos recaudados } \\
\text { hayan sido depositados } \\
\text { de manera íntegra e } \\
\text { intacta en las cuentas } \\
\text { rotativas de ingresos } \\
\text { en el transcurso del } \\
\text { día. }\end{array}$ & $\begin{array}{l}\text { Acta de arqueo } \\
\text { de caja suscrita } \\
\text { por el recaudador } \\
\text { y la persona } \\
\text { designada para el } \\
\text { arqueo. } \\
\text { Comprobantes de } \\
\text { depósito. }\end{array}$ \\
\hline 2 & $\begin{array}{l}\text { Constancia de la } \\
\text { documentación de } \\
\text { recaudación. }\end{array}$ & $\begin{array}{l}\text { Confirmar que los } \\
\text { documentos } \\
\text { generados en la } \\
\text { recaudación de }\end{array}$ & $\begin{array}{l}\text { Verificación que los } \\
\text { comprobantes se } \\
\text { encuentren pre } \\
\text { numerados y sean }\end{array}$ & $\begin{array}{l}\text { Especies } \\
\text { valoradas. } \\
\text { Facturas. }\end{array}$ \\
\hline
\end{tabular}


Revista Interdisciplinaria de Humanidades, Educación, Ciencia y Tecnología

Año V. Vol. V. №1. Edición Especial. 2019

Hecho el depósito de ley: pp201602FA4721

ISSN-L: 2542-3029; ISSN: 2610-802X

Universidad Nacional Experimental Francisco de Miranda (UNEFM). Santa Ana de Coro. Venezuela

Gianna Maritza Apolo Ordoñez; Cecilia Ivonne Narváez Zurita; Juan Carlos Erazo Alvarez

Responsable:

Contador General ingresos cumplan con lo establecido por el SRI. archivados en orden

cronológico.

Evidenciar que los informes de recaudación correspondan a las transacciones realizadas.
Informe suscrito por los recaudadores. Comprobantes de depósito. Copias de títulos de crédito.
1 Cotejar información respecto de la emisión, venta y baja de las especies valoradas. Responsable:

Tesorero

\section{Control y custodia de especies valoradas}

Verificar que el control de las especies valoradas sea llevado mediante cuentas de orden. especies valoradas físicas.
Se realiza el cotejo de información entre las cuentas de orden y las

Revisar que los ingresos generados por la venta de especies valoradas hayan sido depositados en las cuentas rotativas del GAD Municipal de Zaruma.

Elaborado por: Revisado por:

Estados financieros. Archivo de especies valoradas. Bancos Cedulas de ingresos.

Tabla 4

Contabilidad

Gobierno Autónomo Descentralizado Municipal de Zaruma

Procedimientos de control interno

Control de Bancos

№ Procedimiento Objetivo

Verificar la

1 Comprobar que las conciliaciones de los saldos contables conformidad de las operaciones

\section{Acciones de control Evidencia}

Validación de la Registros información registrada. contables. 
CIENCIAMATRIA

Revista Interdisciplinaria de Humanidades, Educación, Ciencia y Tecnología

Año V. Vol. V. №1. Edición Especial. 2019

Hecho el depósito de ley: pp201602FA4721

ISSN-L: 2542-3029; ISSN: 2610-802X

Universidad Nacional Experimental Francisco de Miranda (UNEFM). Santa Ana de Coro. Venezuela

Gianna Maritza Apolo Ordoñez; Cecilia Ivonne Narváez Zurita; Juan Carlos Erazo Alvarez

y bancarios se

realizaron de

manera oportuna.

Responsable:

Director de Gestión

Financiera

1 Verificación de que

los anticipos de

remuneraciones a

servidores han sido

otorgados siguiendo

la normativa.

Responsable:

Director de Gestión

Financiera

2 Evidenciar que los anticipos a contratistas han sido registrados de manera oportuna.

Responsable:

Director de Gestión

Financiera

Elaborado por:

\author{
ejecutadas por la \\ unidad de tesorería. \\ Examinar que los \\ sistemas de carga y \\ registro operen en \\ óptimas condiciones. \\ Estados de \\ cuenta. \\ Informe del \\ técnico de \\ sistemas \\ informáticos.
}

\section{Control de anticipo de fondos}

Comprobar que la entrega de anticipos se realice de acuerdo a lo estipulado por las normas emitidas por las entidades competentes.

Vigilar que los anticipos sean registrados en el sistema.
Se procede a revisar la documentación de solicitud del servidor, autorización y formulario de autorización para el descuento en rol.

Se procede a revisar los estados financieros para constatar el registro de los anticipos.
Solicitud del servidor.

Formulario de autorización.

Roles de pago.

Estados financieros.
Revisado por:

Tabla 5

Deuda pública

Gobierno Autónomo Descentralizado Municipal de Zaruma

Procedimientos de control interno

Control de endeudamiento público

\begin{tabular}{llll}
\hline № & Procedimiento & Objetivo & Acciones de control Evidencia
\end{tabular}


1 Verificación de que la institución no ha superado los límites de endeudamiento previstos en la ley. Responsable: Director de Gestión Financiera, Contadora General

2 Confirmación de presupuesto asignado para asumir el compromiso de crédito. Responsable: Analista de Presupuesto

Elaborado por:

Comprobar que la
institución está
cumpliendo con las
obligaciones de
endeudamiento
contraídas.

Evidenciar que en el presupuesto institucional se han asignado las partidas presupuestarias de desembolsos y asignaciones destinadas a la inversión.

\begin{tabular}{ll}
$\begin{array}{l}\text { Constatar que la } \\
\text { relación porcentual } \\
\text { entre el saldo total de } \\
\text { la deuda y el total de } \\
\text { ingresos no supere el } \\
200 \% \text {. }\end{array}$ & $\begin{array}{l}\text { Estados } \\
\text { financieros. } \\
\text { Centratos de } \\
\text { Cerificar que el servicio } \\
\text { Certificación } \\
\text { presupuestaria. } \\
\text { Informe de la }\end{array}$ \\
$\begin{array}{l}\text { supere el } 25 \% \text { de los } \\
\text { ingresos totales }\end{array}$ & $\begin{array}{l}\text { entidad que } \\
\text { otorga el crédito. }\end{array}$ \\
$\begin{array}{l}\text { anuales. } \\
\text { Se procede a revisar la } \\
\text { cedula presupuestaria } \\
\text { de gastos. }\end{array}$ & $\begin{array}{l}\text { Cedulas } \\
\text { presupuestarias } \\
\text { de gastos. }\end{array}$ \\
$\begin{array}{l}\text { Comprobar que previo } \\
\text { a la suscripción del } \\
\text { contrato de crédito }\end{array}$ & $\begin{array}{l}\text { Certificación } \\
\text { presupuestaria } \\
\text { exista la partida }\end{array}$ \\
$\begin{array}{l}\text { emitida por la } \\
\text { presupuestaria para } \\
\text { asumir el compromiso. } \\
\text { Revisado por: }\end{array}$ & $\begin{array}{l}\text { gestión de } \\
\text { financiera. }\end{array}$ \\
\hline
\end{tabular}

\section{CONCLUSIONES}

El análisis que se ha realizado del control interno como herramienta de apoyo a la gestión financiera del Gobierno Autónomo Descentralizado Municipal de Zaruma con la finalidad de minimizar riesgos y generar información financiera confiable que contribuya a una correcta toma de decisiones financieras ha permitido identificar aspectos importantes de esta técnica como instrumento para el logro de los objetivos institucionales.

Las investigaciones de los autores citados en el presente documento coinciden en varios aspectos del sistema de control interno; de manera específica en que la base del éxito del sistema de control interno es el recurso humano y el compromiso de sus directivos, a esto se suma la importancia que los autores dan a la implementación de un sistema de control interno con procedimientos claros y bien definidos.

Del análisis realizado se pudo observar que las principales deficiencias de control en el departamento de gestión financiera son por el desconocimiento de las funciones que le 


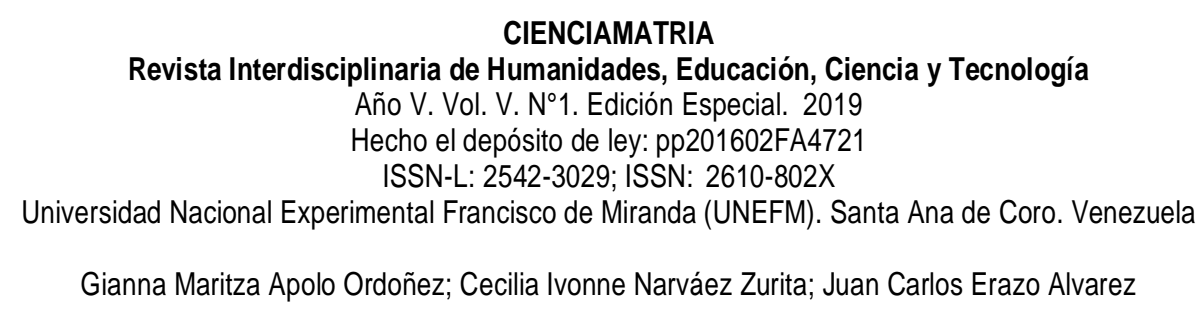

compete a cada uno de los funcionarios, la escaza normativa interna en la que se establezca las responsabilidades y compromisos de los servidores públicos y finalmente no existen procedimientos de control determinados en base a la competencia del personal que sirva de guía para la detección de errores y un correcto control.

La propuesta presentada en este documento está enfocada en definir procedimientos claros y asignar responsabilidades a cada uno de los servidores públicos que desarrollan funciones en el área financiera, además crea un escenario en el que los funcionarios asumen el compromiso de contribuir con el control de cada uno de los procesos como medio para el logro de los objetivos establecidos por la institución.

Se concluye que los procedimientos de control interno permitirán la detección y corrección de errores, además la información que se procese en la empresa tendrá mayor razonabilidad por lo que será integra y confiable, como se menciona en el desarrollo del documento la información contable procesada y reflejada en los estados financieros proporcionan una visión general de la situación económica - financiera que atraviesa la institución, de los estados financiero se recopilará la información más importante para dar un paso hacia la toma de decisiones financieras.

\section{REFERENCIAS CONSULTADAS}

1. Asamblea Constituyente. (2008). Constitución de la República del Ecuador. Quito, Pichincha, Ecuador.

2. Barquero. (2013). Manual Práctico de Control Interno. Barcelona: S.I. Barcelona.

3. Cámara de Diputados. (2014). Marco Integrado de Control Interno. México, D.F.

4. Casals \& Associates, Inc. (2004). Marco integrado de control interno para América Latina. Virginia.

5. Castañeda. (2014). Los sistemas de control interno en las Mipymes y su impacto en la efectividad empresarial. En contexto, 129-146.

6. Contraloría General de la República. (2014). Marco Conceptual del Control Interno. Lima, Perú. 


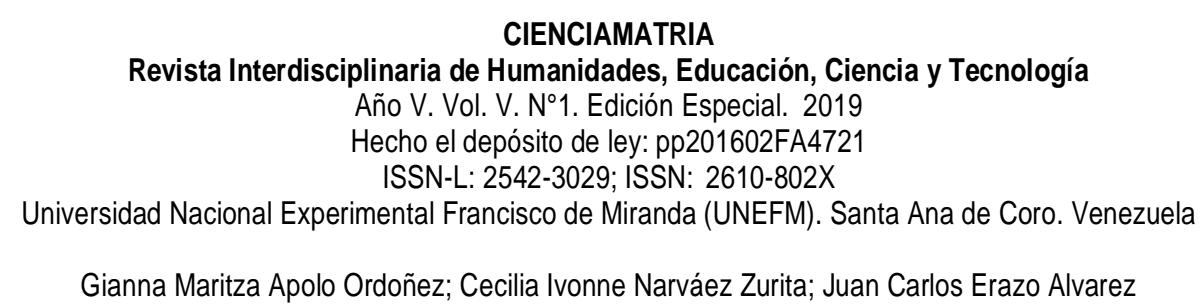

7. Contraloría General del Estado. (2014). Normas de Control Interno. Quito, Pichincha, Ecuador.

8. Córdoba. (2012). Gestión financiera. Bogotá: Ecoe ediciones.

9. Estupiñan. (2015). Control interno y fraudes. Bogotá: Ecoe ediciones.

10. Fonseca. (2011). Sistemas de control interno para organizaciones. Lima: Instituto de Investigación en Accountability y Control - IICO.

11. Hernández, Fernández y Baptista. (2006). Metodología de la Investigación. México.

12. López. (2018). Delitos contra la eficiencia de la administración pública ecuatoriana. Caso de estudio: Morona Santiago. Revista científica de contabilidad, 143-156.

13. Mayorga, \& Llagua. (2018). La evaluación del sistema de control interno como soporte estratégico en la gestión de objetivos en las finanzas populares del Ecuador. Apuntes contables, 117-126.

14. Mendoza. (2013). Gestión financiera pública: Elementos para su estudio y aplicación. Colombia.

15. Ortega Polanco, V. (2017). Gestión de la imagen corporativa de organizaciones universitarias desde el enfoque del marketing emocional. CIENCIAMATRIA, 3(5), 150-171. https://doi.org/10.35381/cm.v3i5.19

16. Pérez, \& Carvallo. (2015). La gestión financiera de la empresa. Madrid: ESIC editorial.

17. Portal. (2016). Control interno e integridad: elementos necesarios para la gobernanza pública. El Cotidiano, 7-13.

18. Romero. (2013). Presupuesto público y contabilidad gubernamental. Bogotá: Ecoe ediciones.

19. Rodríguez Rodríguez, S., Crúz, Y., \& Puertas, A. (2017). Indicadores para la comercialización de productos derivados de lotes caprinos (capra hircus), hacia una estrategia endógena de marketing en Colina - Zamora, Falcón, Venezuela. Revista Arbitrada Interdisciplinaria Koinonía, 2(3), 198--212. Recuperado de http://fundacionkoinonia.com.ve/ojs/index.php/revistakoinonia/article/view/63/50 


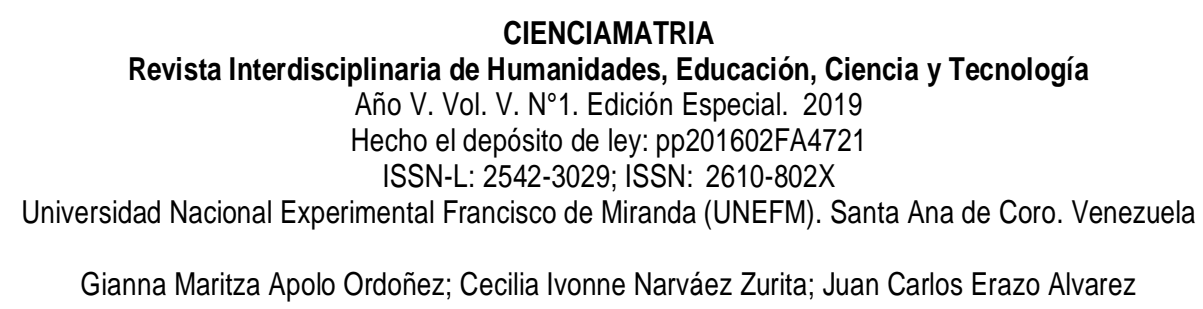

20.Serrano, Señalin, Vega, \& Herrera. (2018). El control interno como herramienta indispensable para una gestión financiera y contable eficiente en las empresas bananeras del cantón Machala (Ecuador). Espacios, 39, 30.

21. Vega. (2016). Control de gestión y control interno: binomio indisoluble de la dirección. Espacios.

22. Vega, \& Ortiz. (07 de Septiembre de 2017). Procesos más relevantes del control interno de una empresa hotelera. Medellín, Colombia.

\section{REFERENCES CONSULTED}

1. Asamblea Constituyente. (2008). Constitution of the Republic of Ecuador. Quito, Pichincha, Ecuador.

2. Barquero. (2013). Practical manual of internal control. Barcelona: S.I. Barcelona.

3. Cámara de Diputados . (2014). Integrated internal control framework. Mexico, Mexi D.F.

4. Casals \& Associates, Inc. (2004). Integrated internal control framework for Latin Amercia. Virginia.

5. Castañeda. (2014). Internal control systems in MSMEs and their impact on business effectiveness. In context, 129-146.

6. Contraloría General de la República. (2014). Conceptual framework of internal control. Lima, Perú.

7. Contraloría General del Estado. (2014). Internal control standards. Quito, Pichincha, Ecuador.

8. Córdoba. (2012). Financial management. Bogota: Ecoe editions.

9. Estupiñan. (2015). Inefficient control and fraud. Bogota: Ecoe editions. 


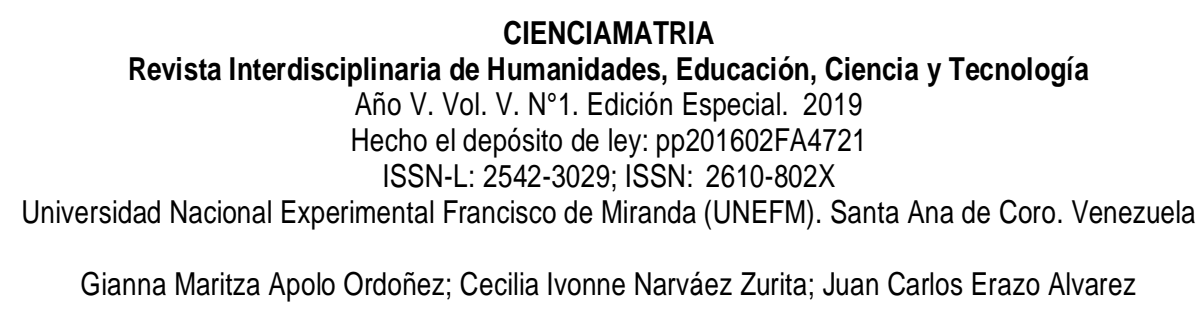

10. Fonseca. (2011). Internal control sistems for organizations. Lima: Investigation institute of accountability and control - IICO.

11. Hernández, Fernández y Baptista. (2006). Investigation methodology. Mexico.

12. López. (2018). Crimes against the efficiency of the Ecuadorian Public administration. Case study: Morona Santiago. Accounting scientific magazine, 143-156.

13. Mayorga, \& Llagua. (2018). The evaluation of the internal control system as a strategic support in the management of objectives in the popular finances of Ecuador. Accounting notes, 117-126.

14. Mendoza. (2013). Public Financial Management: Elements for study and aplication. Colombia.

15. Ortega Polanco, V. (2017). Gestión de la imagen corporativa de organizaciones universitarias desde el enfoque del marketing emocional. CIENCIAMATRIA, 3(5), 150-171. https://doi.org/10.35381/cm.v3i5.19

16. Perez, \& Carvallo. (2015). The financial management of the company. Madrid: ESIC editorial.

17. Portal. (2016). Internal control and integrity : Elements necessary for public governance. The everyday, 7-13.

18. Romero. (2013). Public budget and government accounting. Bogota: Ecoe editions.

19. Rodríguez Rodríguez, S., Crúz, Y., \& Puertas, A. (2017). Indicadores para la comercialización de productos derivados de lotes caprinos (capra hircus), hacia una estrategia endógena de marketing en Colina - Zamora, Falcón, Venezuela. Revista Arbitrada Interdisciplinaria Koinonía, 2(3), 198--212. Recuperado de http://fundacionkoinonia.com.ve/ojs/index.php/revistakoinonia/article/view/63/50

20.Serrano, Señalin, Vega, \& Herrera. (2018). Internal control as an indispensable tool for efficient financial and accounting management in the banana companies of the Machala (Ecuador). Spaces, 39, 30.

21. Vega. (2016). Management control and internal control: indissoluble management binomial. Spaces. 


\section{CIENCIAMATRIA}

Revista Interdisciplinaria de Humanidades, Educación, Ciencia y Tecnología

Año V. Vol. V. №1. Edición Especial. 2019

Hecho el depósito de ley: pp201602FA4721

ISSN-L: 2542-3029; ISSN: 2610-802X

Universidad Nacional Experimental Francisco de Miranda (UNEFM). Santa Ana de Coro. Venezuela

Gianna Maritza Apolo Ordoñez; Cecilia Ivonne Narváez Zurita; Juan Carlos Erazo Alvarez

22. Vega, \& Ortiz. (07 de Septiembre de 2017). Most relevant processes of the internal control of a hotel company. Medellin, Colombia.

(C2019 por los autores. Este artículo es de acceso abierto y distribuido según los términos y condiciones de la licencia Creative Commons Atribución-NoComercial-Compartirlgual 4.0 Internacional (CC BY-NC-

SA 4.0) (https://creativecommons.org/licenses/by-nc-sa/4.0/). 